\title{
A NOTE ON COALGEBRA GAUGE THEORY
}

\author{
TOMASZ BRZEZIŃSKI \\ Department of Applied Mathematics and Theoretical Physics \\ University of Cambridge \\ Cambridge CB3 9EW, U.K. \\ E-mail:T.Brzezinski@damtp.cam.ac.uk
}

\begin{abstract}
A generalisation of quantum principal bundles in which a quantum structure group is replaced by a coalgebra is proposed.

1. Introduction. The main goal of this short note is to summarise a generalisation of the theory of quantum principal bundles of [BM93] in which a quantum structure group is replaced by a coalgebra. The need for such a generalisation arose from the studies of the structure of quantum embeddable homogeneous spaces [Brz95]. This generalisation of gauge theory uses the notion of coalgebra $\psi$-bundles, which was proposed by the author at his lecture delivered at the Banach Center Minisemester on Quantum Groups and Quantum Spaces (November 1995), and then developed in collaboration with S. Majid [BM95]. The examples of $\psi$-bundles include not only the generalised bundles on quantum embeddable homogeneous spaces but also the bundles with braided structure groups. It seems, therefore, that the theory of $\psi$-bundles provides a natural framework for definition and development of braided gauge theories. See Majid's own contribution to these proceedings for more details on braided group gauge theory.

In this article all the algebras are unital and associative, and defined over the field $k$ of characteristic not 2 . In a coalgebra or a Hopf algebra $C, \Delta$ denotes the coproduct written explicitly on elements $c \in C$ as $\Delta c=c_{(1)} \otimes c_{(2)}$, and $\epsilon$ denotes a counit. In a right $C$-comodule $P, \Delta_{R}$ is a right coaction, $\forall u \in P, \Delta_{R} u=u_{(0)} \otimes u_{(1)}$.

This note presents the results obtained in the collaboration with Shahn Majid. Detailed proofs and derivations may be found in [BM95].
\end{abstract}

2. Entwining structures and coalgebra $\psi$-bundles. The following definition is the cornerstone of the construction of coalgebra $\psi$-bundles.

1991 Mathematics Subject Classification: Primary 58B30; Secondary 81R50, 17B37, 16W30. Research supported by the EPSRC grant GR/K02244.

The paper is in final form and no version of it will be published elsewhere. 
Definition 2.1. We say that a coalgebra $C$ and an algebra $P$ are entwined if there is a map $\psi: C \otimes P \rightarrow P \otimes C$ such that

$$
\begin{gathered}
\psi \circ(\mathrm{id} \otimes m)=(m \otimes \mathrm{id}) \circ \psi_{23} \circ \psi_{12}, \quad \psi(c \otimes 1)=1 \otimes c, \quad \forall c \in C \\
(\mathrm{id} \otimes \Delta) \circ \psi=\psi_{12} \circ \psi_{23} \circ(\Delta \otimes \mathrm{id}), \quad(\mathrm{id} \otimes \epsilon) \circ \psi=\epsilon \otimes \mathrm{id},
\end{gathered}
$$

where $m$ denotes multiplication in $P$, and $\psi_{23}=\mathrm{id} \otimes \psi, \psi_{12}=\psi \otimes \mathrm{id}$.

Directly from the definition of the entwining map $\psi$ one derives the following:

Proposition 2.2. Let $C, P$ be entwined by $\psi$. For every group-like element $e \in C$ we have the following:

1. For any positive $n, P^{\otimes n}$ is a right $C$-comodule with the coaction $\Delta_{R}^{n}=\psi_{n n+1} \circ$ $\psi_{n-1 n} \circ \ldots \circ \psi_{12} \circ\left(\eta_{C} \otimes \mathrm{id}^{n}\right) \equiv \overleftarrow{\psi}^{n} \circ\left(\eta_{C} \otimes \mathrm{id}^{n}\right)$, where $\eta_{C}: k \rightarrow C, \alpha \mapsto \alpha e$

2. The coaction $\Delta_{R}^{n}$ restricts to the coaction on $\Omega^{n} P$, where $\Omega^{n} P$ denotes the $P$ bimodule of universal $n-$ forms on P, i.e.

$$
\Omega^{n} P=\left\{\omega \in P^{\otimes n+1} \mid \forall i \in\{1, \ldots, n\}, \quad m_{i i+1} \omega=0\right\},
$$

where $m_{i i+1}$ denotes a multiplication in $P$ acting on the $i$ and $i+1$ factors in $P^{\otimes n+1}$.

3. $M=P_{e}^{c o C}=\left\{u \in P \mid \Delta_{R}^{1} u=u \otimes e\right\}$ is a subalgebra of $P$.

Proposition 2.2 allows us to define the main notion of the coalgebra gauge theory.

Definition 2.3. Let $C, P$ be entwined by $\psi$ and let $e$ be a group-like element in $C$. Let $M=P_{e}^{c o C}$. We say that $P(C, M, \psi, e)$ is a coalgebra $\psi$-bundle over $M$ if the linear map $\chi_{M}: P \otimes_{M} P \rightarrow P \otimes C, u \otimes_{M} v \mapsto u \psi(e \otimes v)$ is a bijection.

ExAmple 2.4. Let $H$ be a Hopf algebra and $P$ be a right $H$-comodule algebra. The linear map $\psi: H \otimes P \rightarrow P \otimes H$ defined by $\psi: c \otimes u \rightarrow u_{(0)} \otimes c u_{(1)}$ entwines $H, P$. Therefore a quantum group principal bundle $P(M, H)$ with universal differential structure as in [BM93] is a $\psi$-principal bundle $P(M, H, \psi, 1)$.

ExAMPLE 2.5. Let $H$ be a Hopf algebra and $\pi: H \rightarrow C$ a coalgebra surjection. If ker $\pi$ is a minimal right ideal containing $\{u-\epsilon(u) \mid u \in M\}$ then $\psi: C \otimes H \rightarrow H \otimes C$ defined by $\psi(c \otimes u)=u_{(1)} \otimes \pi\left(v u_{(2)}\right)$ entwines $C, H$, where $u \in H, c \in C$ and $v \in \pi^{-1}(c)$, and we have a $\psi$-principal bundle $H(M, C, \psi, \pi(1))$. Hence the generalised bundles over embeddable quantum homogeneous spaces in [Brz95] are examples of $\psi$-principal bundles.

EXAMPLE 2.6. Let $B$ be a braided group with braiding $\Psi$ (cf [Maj93]) and $P$ a right braided $B$-comodule algebra. The linear map $\psi: B \otimes P \rightarrow P \otimes B$ defined by $\psi: c \otimes u \rightarrow \Psi\left(c \otimes u_{(0)}\right) u_{(1)}$ entwines $B, P$. If the induced map $\chi_{M}$ is a bijection we say that the associated $\psi$-principal bundle $P(M, B, \psi, 1)$ is a braided group principal bundle, and denote it by $P(M, B, \Psi)$.

Example 2.7. Let $P$ and $C$ be entwined by $\psi$ as in Definition 2.1 and let $e$ be a group-like element in $C$. Assume the following data:

1. A map $\psi^{C}: C \otimes C \rightarrow C \otimes C$ such that

$$
(\mathrm{id} \otimes \Delta) \circ \psi^{C}=\psi_{12}^{C} \circ \psi_{23}^{C} \circ(\Delta \otimes \mathrm{id}), \quad(\mathrm{id} \otimes \epsilon) \circ \psi^{C}=\epsilon \otimes \mathrm{id},
$$

and $\psi^{C}(e \otimes c)=\Delta c$, for any $c \in C$; 
2. A convolution invertible map $\Phi: C \rightarrow P$ such that $\Phi(e)=1$ and

$$
\psi \circ(\mathrm{id} \otimes \Phi)=(\Phi \otimes \mathrm{id}) \circ \psi^{C} \text {. }
$$

Then there is a $\psi$-principal bundle over $M=P_{e}^{c o C}$. This bundle is called a trivial $\psi$-principal bundle and is denoted by $P\left(M, C, \Phi, \psi, \psi^{C}, e\right) . \quad P$ is also an example of a crossed product of $M$ by a coalgebra $C$ (cf. [Brz96]).

In the finite dimensional case the definition of an entwining structure $\psi: C \otimes P \rightarrow$ $P \otimes C$ is equivalent to the definition of an algebra factorisation structure $\tilde{\psi}: C^{*} \otimes P^{\text {op }} \rightarrow$ $P^{\mathrm{op}} \otimes C^{*}$ which plays an important role in the theory of Hopf algebra double cross products [Maj90]. Finally we remark that Definition 2.1 is self-dual in the following sense: The conditions of Definition 2.1 remain the same if we replace $\Delta$ with $m$ and then reverse the order of the composition of maps. Thus we have the following

Proposition 2.8. Let $C, P$ be entwined by $\psi: C \otimes P \rightarrow P \otimes C$. For every algebra character $\kappa: P \rightarrow k$ we have the following:

1. For any positive integer $n, C^{\otimes n}$ is a right $P$-module with the action $\triangleleft^{n}=(\kappa \otimes$ $\left.\mathrm{id}^{n}\right) \circ \psi_{12} \circ \psi_{23} \circ \ldots \circ \psi_{n n+1}=\left(\kappa \otimes \mathrm{id}^{n}\right) \circ \vec{\psi}^{n}$.

2. The action $\triangleleft^{n}$ maps $\Delta^{n}(C)$ to itself.

3. The subspace $I_{\kappa}=\operatorname{span}\left\{c \triangleleft^{1} u-c \kappa(u) \mid c \in C, u \in P\right\}$ is a coideal. Hence $M=C / I_{\kappa}$ is a coalgebra. We denote the canonical projection by $\pi_{\kappa}: C \rightarrow M$.

4. There is a map $\zeta^{M}: C \otimes P \rightarrow C \otimes{ }^{M} C$ defined by $\zeta^{M}(c \otimes u)=c_{(1)} \otimes c_{(2)} \triangleleft^{1} u$, where $C \otimes{ }^{M} C=\operatorname{span}\left\{c \otimes d \in C \otimes C \mid c_{(1)} \otimes \pi_{\kappa}\left(c_{(2)}\right) \otimes d=c \otimes \pi_{\kappa}\left(d_{(1)}\right) \otimes d_{(2)}\right\}$ is the cotensor product under $M$. If $\zeta^{M}$ is a bijection, we say that $C(M, P, \psi, \kappa)$ is a dual $\psi$-principal bundle.

3. Connections in $\psi$-bundles. If $C$ and $P$ are entwined by $\psi$ then the universal differential envelope of $P$ is $C$-covariant, i.e. the coaction $\Delta_{R}^{n}$ restricts to $\Omega^{n} P$, by the second assertion of Proposition 2.2, and $\overleftarrow{\psi}^{n} \circ(\mathrm{id} \otimes \mathrm{d})=(\mathrm{d} \otimes \mathrm{id}) \circ \overleftarrow{\psi}^{n-1}$. Here d denotes the natural extension of the Karoubi differential $\mathrm{d} u=1 \otimes u-u \otimes 1$ to higher forms.

Definition 3.1 Let $P(M, C, \psi, e)$ be a generalised quantum principal bundle such that $\psi(C \otimes M) \subset M \otimes C$. A connection in $P(M, C, \psi, e)$ is a left $P$-module projection $\Pi: \Omega^{1} P \rightarrow \Omega^{1} P$ such that $\operatorname{ker} \Pi=P \Omega^{1} M P$ and $\overleftarrow{\psi}^{2}(\mathrm{id} \otimes \Pi)=(\Pi \otimes$ id $) \overleftarrow{\psi}^{2}$

The assumption $\psi(C \otimes M) \subset M \otimes C$ ensures that $\overleftarrow{\psi}^{2}\left(C \otimes P \Omega^{1} M P\right) \subset P \Omega^{1} M P \otimes C$ and thus the requirement that $\Pi$ be covariant is compatible with the requirement on the kernel of $\Pi$.

Equivalently we can describe a connection $\Pi$ as follows. We define a map $\phi: C \otimes P \otimes$ $\operatorname{ker} \epsilon \rightarrow P \otimes \operatorname{ker} \epsilon \otimes C$ by

$$
\phi \circ(\mathrm{id} \otimes \chi)=(\chi \otimes \mathrm{id}) \circ \overleftarrow{\psi}^{2}
$$

where $\chi(u \otimes v)=u \psi(e \otimes v)$. By definition of $P(M, C, \psi, e)$ we have a short exact sequence of left $P$-module maps

$$
0 \rightarrow P \Omega^{1} M P \rightarrow \Omega^{1} P \stackrel{x}{\rightarrow} P \otimes \operatorname{ker} \epsilon \rightarrow 0 .
$$


Proposition 3.2 The existence of a connection $\Pi$ in $P(M, C, \psi, e)$ is equivalent to the existence of a left $P$-module splitting $\sigma: P \otimes \operatorname{ker} \epsilon \rightarrow \Omega^{1} P$ of the above sequence such that $\overleftarrow{\psi}^{2} \circ(\mathrm{id} \otimes \sigma)=(\sigma \otimes \mathrm{id}) \circ \phi$

A simple consequence of Proposition 3.2 is the fact that to each connection we can associate its connection one-form $\omega: \operatorname{ker} \epsilon \rightarrow \Omega^{1} P$ by setting $\omega(c)=\sigma(1 \otimes c)$. Clearly $\chi \circ \omega(c)=1 \otimes c$. Also, $\omega$ has certain covariance properties coming from the fact that $\sigma$ intertwines $\overleftarrow{\psi}^{2}$ with $\phi$. These properties generalise the usual right-adjoint covariance of $\omega$. We do not display them explicitly here (cf. Proposition 3.5 of [BM95] for details) but only remark that they characterise $\omega$ completely. Any map $\omega: \operatorname{ker} \epsilon \rightarrow \Omega^{1} P$ having those properties and such that $\chi \circ \omega(c)=1 \otimes c$ gives the unique connection $\Pi=m \circ(\mathrm{id} \otimes \omega) \circ \chi$ in $P(M, C, \psi, e)$. For example, in a trivial $\psi$-bundle $P\left(M, C, \Phi, \psi, \psi^{C}, e\right)$ as in Example 2.7. one can always define a trivial connection one-form by $\omega(c)=\Phi^{-1}\left(c_{(1)}\right) \mathrm{d} \Phi\left(c_{(2)}\right)$, provided that $\psi(M \otimes C) \subset M \otimes C$. Finally, we also remark that the covariance properties of $\omega$ reduce to the covariance of $\omega$ under the braided right-adjoint coaction in the case of the braided group principal bundle $P(M, B, \Psi)$ of Example 2.6.

4. Outlook. The theory of coalgebra $\psi$-bundles proposed in [BM95] and summarised in the present note opens new possibilities for developing quantum group gauge theories. The most urgent and, probably, the most challenging problem, already being investigated, is to obtain a geometric construction of braided group gauge theories. The original approach to quantum group gauge theories of [BM93] suffered from the lack of comprehensive description of local structure of gauge theories in terms of Lie algebras. It is now hoped that in the case of braided groups, for which the notion of a braided Lie algebra is well-known [Maj94], one can overcome this difficulty and successfully use braided gauge theory in situations of truly physical interest.

\section{References}

[Brz95] T. Brzeziński, Quantum Homogeneous Spaces as Quantum Quotient Spaces, J. Math. Phys. 37 (1996) 2388.

[Brz96] T. Brzeziński, Crossed Products by a Coalgebra, Preprint DAMTP/96-28 (1996), q-alg/9603016.

[BM93] T. Brzeziński and S. Majid, Quantum Group Gauge Theory on Quantum Spaces, Commun. Math. Phys. 157 (1993) 591; it ibid. 167 (1995) 235 (erratum).

[BM95] T. Brzeziński and S. Majid, Coalgebra Gauge Theory, Preprint DAMTP/95-74, (1995), q-alg/9602022.

[Maj90] S. Majid, Physics for algebraists: Non-commutative and non-cocommutative Hopf algebras by a bicrossproduct construction, J.Algebra, 130 (1990) 17.

[Maj93] S. Majid, Beyond supersymmetry and quantum symmetry (an introduction to braided groups and braided matrices), In: M.L. Ge and H.J. de Vega, editors, Quantum Groups, Integrable Statistical Models and Knot Theory, 231-238, World Scientific, 1993.

[Maj95] S. Majid, Quantum and braided Lie algebras, J. Geom. Phys. 13(1994)307. 